Article

\title{
Genome-Wide Analysis Reveals Dynamic Epigenomic Differences in Soybean Response to Low-Phosphorus Stress
}

\author{
Shanshan Chu ${ }^{1}$, Xiangqian Zhang ${ }^{1}$, Kaiye Yu ${ }^{1}$, Lingling Lv ${ }^{1}$, Chongyuan Sun ${ }^{1}$, Xiaoqian Liu ${ }^{1}$, \\ Jinyu Zhang ${ }^{2}$, Yongqing Jiao ${ }^{1}$ and Dan Zhang ${ }^{1, * \mathbb{C}}$ \\ 1 Collaborative Innovation Center of Henan Grain Crops, College of Agronomy, \\ Henan Agricultural University, Zhengzhou 450046, China; chushan3@163.com (S.C.); \\ 15333717403@163.com (X.Z.); 17638110601@163.com (K.Y.); 13525606625@163.com (L.L.); \\ 13183019220@163.com (C.S.); liuxq2019@126.com (X.L.); jiaoyongqing@henau.edu.cn (Y.J.) \\ 2 Collaborative Innovation Center of Modern Biological Breeding, Henan Institute of Science and Technology, \\ Xinxiang 453003, China; zjy891@126.com \\ * Correspondence: zhangd@henau.edu.cn; Tel.: +86-371-5699-0186
}

Received: 1 August 2020; Accepted: 15 September 2020; Published: 17 September 2020

\begin{abstract}
Low-phosphorus (low-P) stress has a significant limiting effect on crop yield and quality. Although the molecular mechanisms of the transcriptional level responsible for the low-P stress response have been studied in detail, the underlying epigenetic mechanisms in gene regulation remain largely unknown. In this study, we evaluated the changes in DNA methylation, gene expression and small interfering RNAs (siRNAs) abundance genome-wide in response to low-P stress in two representative soybean genotypes with different P-efficiencies. The DNA methylation levels were slightly higher under low-P stress in both genotypes. Integrative methylation and transcription analysis suggested a complex regulatory relationship between DNA methylation and gene expression that may be associated with the type, region, and extent of methylation. Association analysis of low-P-induced differential methylation and gene expression showed that transcriptional alterations of a small part of genes were associated with methylation changes. Dynamic methylation alterations in transposable element (TE) regions in the $\mathrm{CHH}$ methylation context correspond with changes in the amount of siRNA under low-P conditions, indicating an important role of siRNAs in modulating TE activity by guiding $\mathrm{CHH}$ methylation in TE regions. Together, these results could help to elucidate the epigenetic regulation mechanisms governing the responses of plants to abiotic stresses.
\end{abstract}

Keywords: DNA methylation; epigenetics; low-phosphorus stress; gene expression; small RNA; soybean

\section{Introduction}

The processes of plant growth and development are generally subject to various environmental stresses, including biotic and abiotic stress. During evolution, plants gradually evolved elaborate sensory and adaptive mechanisms, including changes at the physiological and biochemical levels, to better adapt to adverse environmental conditions [1]. Recent studies have identified a large number of genes encoding transcriptional factor regulation of gene transcription, enzymes involved in stress signal transduction, and functional proteins that change downstream cell status participating in plant stress responses [2]. Furthermore, epigenetic regulation factors have been suggested to play an important role in the transcriptional and posttranscriptional control of these genes [3]. DNA methylation is one of the most well-studied epigenetic markers $[4,5]$ that modulate gene expression in response to both biotic and abiotic stresses [6,7]. 
DNA methylation exists in three sequence contexts, including CG, CHG, and $\mathrm{CHH}$ (where $\mathrm{H}=\mathrm{A}, \mathrm{C}$, or T), by activating different DNA methyltransferase enzymes and the RNA-directed DNA methylation (RdDM) pathway in plants [8-10]. DNA methylation in the symmetric CG and CHG contexts is copied during DNA replication and established by conserved methyltransferase1 (MET1) and the plant-specific DNA methyltransferase chromomethylase 3 (CMT3), respectively [5,11-13]. DNA methylation in the nonsymmetrical $\mathrm{CHH}$ context is generated de novo after DNA replication and established by the small (typically 24 nucleotides) interfering RNA-directed DNA methylation (RdDM) pathway [3]. The extent of genomic DNA methylation is maintained not only by the DNA methylation process but also by the DNA demethylation process catalyzed by several DNA demethylases, such as the DEMETER family $[14,15]$.

Previous investigations on the alterations of DNA methylation coping with stresses have utilized low-resolution and nonquantitative methods [16-19]. Nevertheless, the emergence of high-throughput genomic sequencing technology enables single-base-resolution analysis of DNA methylation in the genome-wide range [20,21], thereby enabling global assessment of the pattern changes of DNA methylation responding to various environmental cues. Numerous studies have shown that environmental stress on plants could significantly induce changes in methylation levels in genes companied with changes in transcriptional abundance. In poplar (Populus trichocarpa), drought stress induced widespread alterations in DNA methylation [22]. Moreover, the extent of changes in genomic DNA methylation has affected abundant drought-related transcriptional changes [23]. Additionally, environmental stresses also changed epigenetic variations in transposable element (TE), indicating that TEs are involved in the plant stress response with epigenetic alterations [7]. For instance, a Tam3 transposon methylation alteration at $\mathrm{CHH}$ sites in snapdragon (Antirrhinum majus) was detected in response to low-temperature stress [24]. In addition, the Mutator element MuDR was demethylated along with the increased expression level of the mudrA transposase gene in maize responding to low nitrogen ion stress [25]. These investigations revealed that environmental stresses could generate significant effects on DNA methylation alterations and TE mobilization.

In natural and agricultural ecosystems, one of the most common abiotic stresses is low phosphorus (P) availability [26,27], which restricts crop productivity in more than $70 \%$ of globally available arable land [28]. To overcome the issues of low availability of inorganic $P$ in the soil [29], applying a large amount of $\mathrm{P}$ fertilizer has become the main strategy to maintain crop yield. However, excessive $\mathrm{P}$ application not only increases the input-output ratio but also causes the accumulation of harmful elements in the soil and environmental pollution. Even more worrying is that phosphorus resources are not renewable, and the world's available phosphate mines will be depleted in the next $50-80$ years at current mining rates [27]. Therefore, a better understanding of the molecular mechanism involved in P homeostasis to improve the absorption and utilization efficiency of $\mathrm{P}$ in crops is a sustainable way to improve global food security.

Plants have evolved a range of sophisticated responses aimed at coping with low $\mathrm{P}$ availability [2,30]. In low-P stress, the primary root is responsible for sensing local low-P signals [31], and transcription factors, such as PHR1 and PHL1, are responsible for modulating long-distance phosphate signaling in Arabidopsis thaliana [32]. Both PT genes encoding high-affinity phosphate transporters and $A C P$ genes encoding acid phosphatase could increase phosphate uptake by improving expression levels. In addition, SPX-domain-containing proteins, such as SPX and PHO1, have been reported to mediate the regulation of phosphorus homeostasis [30,33-38].

Despite the considerable advances in understanding transcriptional and posttranscriptional mechanisms of plant responses to low $\mathrm{P}$ availability, several epigenetic mechanisms regulating gene expression coping with this stress have only been assessed by a limited number of studies that are confined to few model organisms [39-41]. For example, in Arabidopsis, global DNA methylation occurs with extensive remodeling under low-P stress, which is associated with changes in P starvation response gene expression. This study revealed that dynamic methylation changes play pivotal roles in response to P starvation [40]. Soybean is the main source of human edible oils and vegetable proteins. 
Compared with nonlegumes, such as rice or corn, soybean requires more $\mathrm{P}$ because of the higher $\mathrm{P}$ content in soybean seeds [42]. P deficiency in soybeans not only affects the growth and development of plants and increases the loss of flower, as well as pods, but also affects the formation of nodules, thereby reducing nitrogen fixation efficiency and ultimately affecting its quality and yield [43-45]. Therefore, soil P deficiency has become an important factor limiting the development of soybean production [46]. Accordingly, it is particularly important to understand the epigenetic regulation mechanism of the low-P stress response in soybean.

In our study, we constructed DNA methylation maps with single-base resolution and genome-wide coverage in two representative soybean genotypes with different $P$ efficiencies, 'Nannong 94156 ' and 'Bogao' (a tolerant genotype and a sensitive genotype to low-P stress, respectively) under low-P $(\mathrm{LP},-\mathrm{P}, 5 \mu \mathrm{M})$ and high-P $(\mathrm{HP},+\mathrm{P}$, control, $500 \mu \mathrm{M})$ conditions, respectively. This investigation was designed to answer two main questions: (i) the genomic landscape and changes in the soybean methylome associated with low-P stress (ii) and the relationship between methylome alterations and P-efficiency-associated gene expression alterations.

\section{Results}

\subsection{Genome-Wide DNA Methylation Patterns in Response to Low-P Stress}

To understand the genomic DNA methylation features and patterns at a single nucleotide in response to $\mathrm{P}$ availability, we examined the global DNA methylation levels in root tissues of the representative low-P-tolerant ' $\mathrm{NN}$ ' and low-P-sensitive 'BG' cultivars by single-base resolution whole-genome bisulfite sequencing [5]. In total, our bisulfite sequencing yielded 366193864-522038932 raw reads for each of the four DNA library samples (Table 1). After removal of adapter contaminants, low-quality reads and reads containing Ns, 360336480-515810512 clean reads were collected (Table 1), of which approximately $88 \%$ were mapped into the soybean genome. Moreover, approximately $67 \%$ of cytosines were covered by more than one uniquely mapped read in the soybean genome. The sequencing data were ready for further analysis, while the sequencing depth reached $50 \times$, and the detected cytosine number reached saturation (Figure S1). Bisulfite conversion efficiency ranged from $99.62 \%$ to $99.66 \%$ per sample, as determined using the nonmethylated $\lambda$ phage genome (Table 1 ).

Table 1. Summary of genome-wide methylation sequencing data.

\begin{tabular}{ccccccc}
\hline Sample & $\begin{array}{c}\text { Raw } \\
\text { Reads }\end{array}$ & $\begin{array}{c}\text { Clean } \\
\text { Reads }\end{array}$ & $\begin{array}{c}\text { Mapped } \\
\text { Reads }\end{array}$ & $\begin{array}{c}\text { Mapped } \\
\text { Ratio (\%) }\end{array}$ & $\begin{array}{c}\text { Sequence } \\
\text { Depth }\end{array}$ & $\begin{array}{c}\text { Bisulfite Conversion } \\
\text { Efficiency Ratio (\%) }\end{array}$ \\
\hline NN_HP & 444676244 & 438763364 & 387477437 & $88.31 \%$ & 59.40 & $99.62 \%$ \\
NN_LP & 366193864 & 360336480 & 316853049 & $87.93 \%$ & 48.57 & $99.64 \%$ \\
BG_HP & 522038932 & 515810512 & 458006046 & $88.79 \%$ & 70.21 & $99.65 \%$ \\
BG_LP & 404607624 & 398836454 & 350842529 & $87.97 \%$ & 53.78 & $99.66 \%$ \\
\hline
\end{tabular}

NN_HP represents 'Nan-nong94-156' under control conditions; NN_LP represents 'Nan-nong94-156' under low-P conditions; BG_HP represents 'Bogao' under control conditions, and BG_LP represents 'Bogao' under low-P stress.

The NN_HP ('Nan-nong94-156'_high P) genome presented 66.50\% mCG (mCG/CG), 43.63\% $\mathrm{mCHG}(\mathrm{mCHG} / \mathrm{CHG})$, and $3.68 \% \mathrm{mCHH}(\mathrm{mCHH} / \mathrm{CHH})$, which showed the percentage of methylation levels in the soybean genome. Correspondingly, BG_HP ('Bogao'_high P) presented 67.48\%, 44.00\%, and $3.84 \%$ in CG, CHG, and CHH contexts, respectively (Table S1 and Figure S2). We found that 'NN' and 'BG' exhibit a similar pattern in response to P availability (LP vs. HP) and that all three sequence contexts of DNA methylation levels were slightly higher after low-P stress. While investigating the distributions of $\mathrm{mCs}$ in three sequence contexts, we observed that methylcytosine was most common at the $\mathrm{CHH}$ sites (40.4-42.8\%) and occurred less frequently in CG and CHG sequences $(30.1-31.6 \%$ and 27.2-28.0\%, respectively). A slight increase in CG and CHG methylation proportions and a decrease in $\mathrm{CHH}$ methylation was found in both 'NN' and 'BG' under low-P stress (Figure 1a). Global DNA methylation profiles demonstrated that a high degree of methylation occurred in transposable element (TE)-rich regions, while the gene-rich regions exhibited relatively reduced methylation in the soybean 
genome (Figure 1b). This result was similar to previous findings in soybean [47], Arabidopsis [48], and rice [49], suggesting that DNA methylation in transposon silencing might be conserved in plants.

a
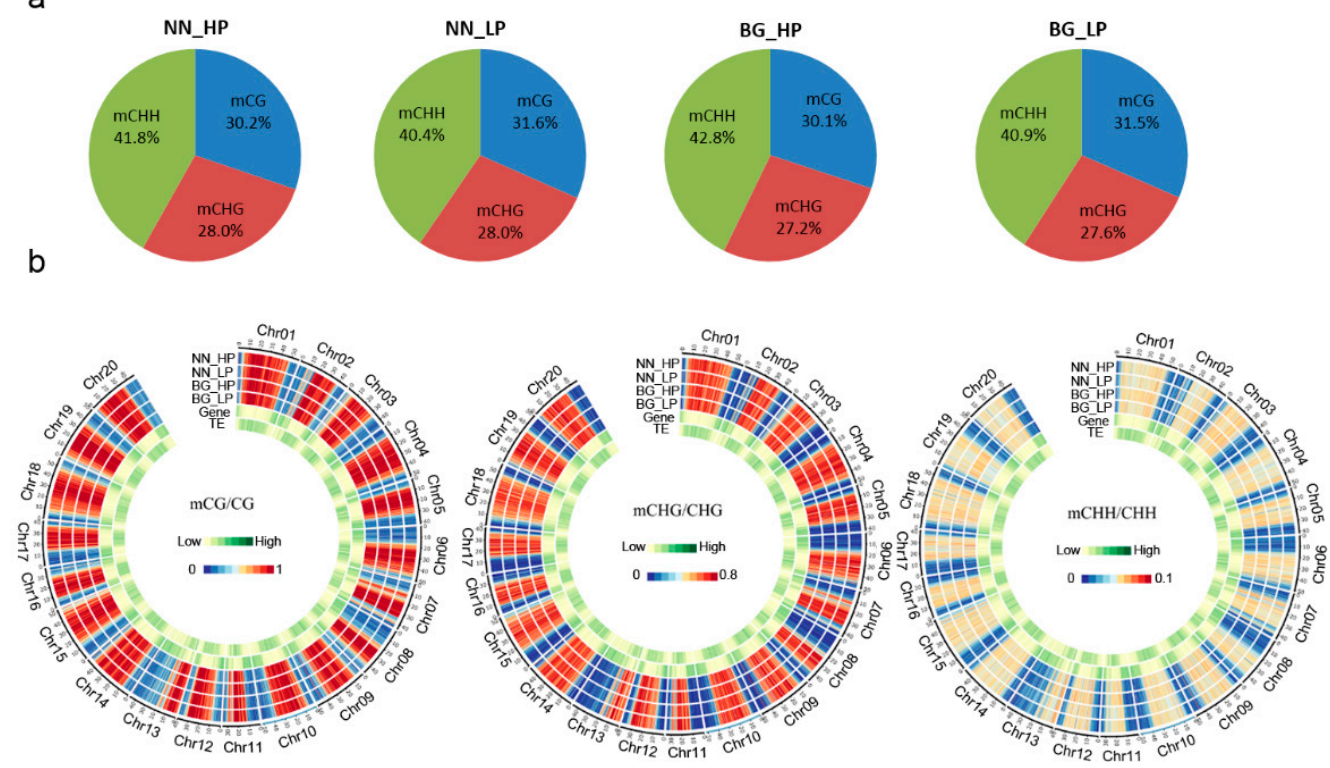

Figure 1. DNA methylome features in soybean. (a) Relative proportions of $\mathrm{mCs}$ in three sequence contexts (CG, CHG, and $\mathrm{CHH}$ ); (b) A circos plot of gene and transposon density and $\mathrm{mCG}, \mathrm{mCHG}$, and $\mathrm{mCHH}$ location in soybean. NN_HP represents 'Nan-nong94-156' under control conditions; NN_LP represents 'Nan-nong94-156' under low-P conditions; BG_HP represents 'Bogao' under control conditions, and BG_LP represents 'Bogao' under low-P stress.

\subsection{DNA Methylation Patterns in Gene and TE Regions}

While inspecting the distribution of CG, $\mathrm{CHG}$, and $\mathrm{CHH}$ methylations in gene and TE regions, we observed that CG methylation occurred preferentially in the gene body regions relative to the flanking regions, similar to previous reports in other plants $[47,48,50,51]$, whereas the extents of CHG and CHH methylation were low in gene body regions and relatively higher in flanking regions (Figure 2a). The DNA methylation extents of the CG, CHG, and CHH contexts were notably low near transcriptional start sites and transcriptional end sites but increased gradually with increasing distance from these sites (Figure 2a). In contrast to gene body regions, the TEs were highly methylated in all CG, $\mathrm{CHG}$, and $\mathrm{CHH}$ sequence contexts (Figure 2b). Furthermore, we noted that most of the methylated TEs belonged to class I (retro-transposons), especially for LTR/Gypsy and LTR/Copia, consistent with their abundances in the soybean genome. Among class II (DNA transposons), the TE-type DNA/MuDR was more frequently methylated than others (Figure S3a).

Unsurprisingly, the low-P treatment clearly exhibited CG, CHG, and CHH hypermethylation in both the gene body and flanking regions compared with the high phosphorus treatment (Figure 2a). In TE regions, striking differences of methylation levels were observed in the $\mathrm{CHH}$ methylation context, while no significant differences were observed in CG and CHG methylation contexts between different P levels treatments. The NN_LP exhibited a lower CHH methylation level in TE regions than in the NN_HP (Figure 2b). Consistent with the similar methylation extent of TEs in both phosphorus treatments, the average methylation extents of both class I and II TEs are largely the same in CG and CHG contexts between different phosphorus treatments. The TEs within class I exhibited a higher methylation extent than those within class II in CG and CHG contexts, whereas the CHH methylation extent of the class II TEs appeared to be significantly higher compared with the class I TEs (Figure S3b). Interestingly, in NN_LP, the reduced CHH methylation extent of TEs may be due to the lower average methylation level of class I TE (Figure S3b). 
a
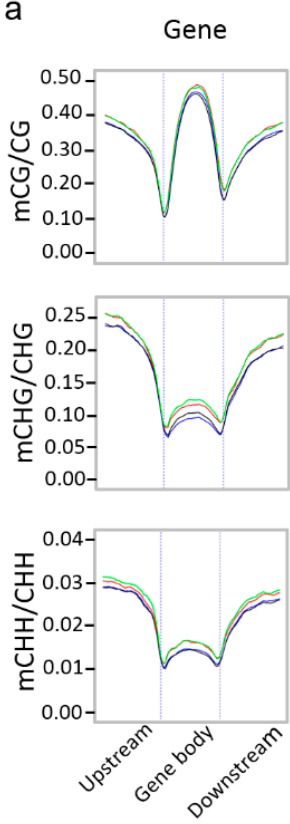

-NN_HP - NN_LP b
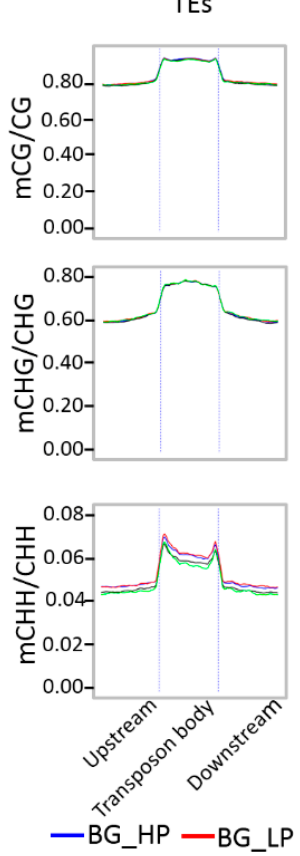

Figure 2. Genomic DNA methylation profiles in soybean. (a) DNA methylation patterns across genes; (b) DNA methylation patterns across TEs; the vertical dashed line represents the boundaries of the gene body or transposon (TE) body.

\subsection{Association Analyses of DNA Methylation Status and Gene Expression}

DNA methylation controls genes in numerous biological processes. To unveil how the promoter and gene body methylation functions in gene expression, transcriptome profiles of low-P-treated ' $\mathrm{NN}$ ' and 'BG' were generated. These materials are identical to the ones for methylome analysis. Genes were separated into two parts, the non-expressed (none) genes (FPKM value $<0.1$ ) and the expressed genes. Based on the expression level, the expressed genes were further divided into four groups in ascending order. Non-expressed genes maintained relatively higher methylation levels as expected in all three sequence contexts (Figure 3a). Correspondingly, expressed genes with the highest expression levels had the lowest CG and CHG methylation levels in gene body and flanking regions, and the lowest $\mathrm{CHH}$ methylation levels in gene body regions (Figure 3a). The moderately expressed genes had moderate CG, CHG, and CHH methylation levels in gene body regions. However, the expressed genes with low or moderate expression levels showed lower $\mathrm{CHH}$ methylation levels than the highly expressed genes in promoter and downstream 2-kb regions (Figure 3a).

For further study of the connection between gene methylation and expression, genes were categorized as methylated or unmethylated according to the methylation level. Methylated genes were ranked based on the promoter or gene body methylation levels. Then, the genes were sorted into five groups accordingly (Figure $3 \mathrm{~b}$ ). The first $20 \%$ was the genes involved with the lowest methylation level, while the fifth group was the highest. The Figure $3 \mathrm{~b}$ shows that there is an inverse correlation between promoter methylation and gene expression, since higher levels of promoter methylation show lower expression levels. Consistent with these results, the gene body with the highest methylation levels showed the lowest expression levels, but there was no significant difference among the other methylation-level groups. In addition, we performed a Spearman correlation analysis between methylation and gene expression levels. As shown in Figure S4, the overall correlation rho was low regardless of the methylation types. However, the rho can reach -0.22 in downstream $2-\mathrm{kb}$ regions for CG methylation. This result suggested that methylation levels in a small fraction of downstream 2-kb regions have relatively higher correlation with their expression levels. 
a

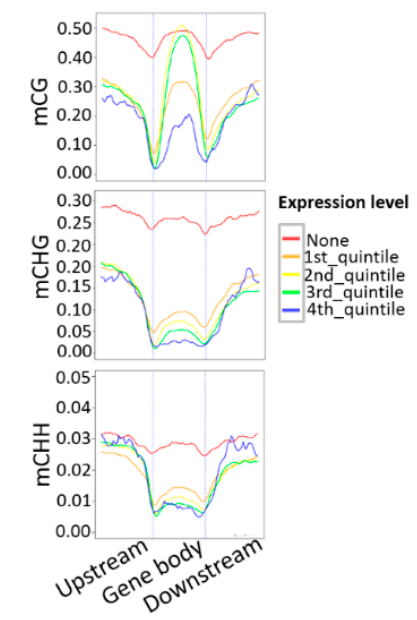

b

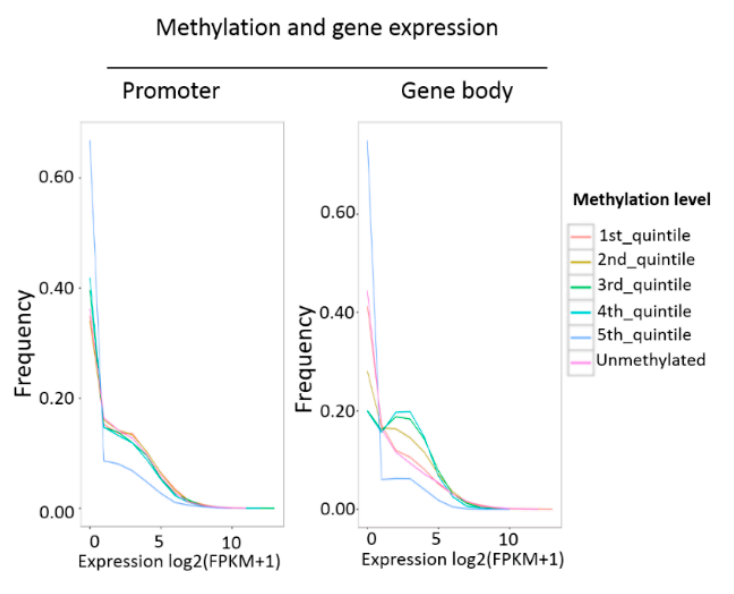

Figure 3. Relationship between DNA methylation and gene expression. (a) Distributions of methylation levels within gene bodies partitioned by different expression levels: 1st_quintile is the lowest and 4th_quintile is the highest; genes with FPKM value $<0.1$ were considered non-expressed (none); (b) expression profiles of methylated genes compared with unmethylated genes. Methylated genes were further divided into quintiles based on promoter and gene body region methylation levels: 1st_quintile is the lowest and 5th_quintile is the highest.

\subsection{Identification of DMR and DMR-Associated Genes in Response to Low-P Stress}

To explore the possible influence of low-P stress on methylation, we identified the differentially methylated regions (DMRs) between NN_LP vs NN_HP and BG_LP vs BG_HP ('NN' low-P versus high-P and ' $\mathrm{BG}$ ' low-P versus high-P) by comparing fractional methylation levels of $10 \mathrm{~kb}$ windows throughout the genome (FDR < 0.05). In response to low-P stress, we found more low-P-inducible hyper-DMRs (i.e., higher methylation in low-P-treated accessions) in all contexts in both ' $\mathrm{NN}^{\prime}$ and 'BG' and CHH-DMRs were most abundant among all methylation contexts (Figure S5). The cluster analysis also revealed a widespread methylome change under low-P stress (Figure S6). Among these CG DMRs, approximately 46-49\% of DMRs were located in TE regions, 31-34\% of DMRs were located in the gene body region, while only $10 \%$ of DMRs were located in 2-kb upstream and downstream regions of the gene, respectively. Similarly, most CHG DMRs (approximately 60\%) were found in TE regions, $17-18 \%$ of DMRs were found in the gene body region, and only $11-12 \%$ of DMRs were found in upstream and downstream regions, respectively. For the CHH DMRs, most of them (approximately $41-45 \%$ ) were also located in TE regions, $21-27 \%$ were located in the upstream or downstream regions, while only $11 \%$ of DMRs were located in the gene body region (Figure S7). Briefly, most DMRs in all three contexts were found in the TE regions. These analyses provided a clear landscape of genomic methylation differentiation in BG_LP vs BG_HP and NN_LP vs NN_HP.

Some overlap between DMRs and genes was detected based on the association between the DMR positions with protein-coding genes and $2 \mathrm{~kb}$ upstream and downstream regions. In total, 7132 hypermethylated and 4361 hypomethylated genes were identified in NN_LP vs NN_HP; similarly, 8581 hypermethylated genes and 5015 hypomethylated genes were identified in BG_LP vs BG_HP (Table S2). 'NN' and 'BG' shared 2363 hypermethylated genes and 856 hypomethylated genes under low-P stress (Figure 4a). To obtain a deeper understanding of the potential biological functions of DMR genes, a Gene Ontology (GO) category analysis was performed. We found that these DMR genes were primarily associated with nucleic acid, phosphorus, and nitrogen compound metabolic processes, stressing response, macromolecular complex, and ion binding, regardless of whether they are hypermethylated genes or hypomethylated genes (Figure S8). Furthermore, we identified pathways affected by low-P treatment in both genotypes using KEGG (Kyoto Encyclopedia of Genes and 
Genomes) pathway enrichment analysis. As shown in Figure 4b, hypermethylated genes in NN_LP vs NN_HP show abundant enrichment in pathways related to such processes as nucleotide excision repair, oxidative phosphorylation, spliceosome, and RNA transport. The last two pathways are also enriched in BG_LP vs BG_HP. Among hypomethylated genes in NN_LP vs NN_HP, pathways of RNA degradation, carbon metabolism, and plant hormone signal transduction were enriched. In addition to the last pathway, hypomethylated genes in BG_LP vs BG_HP are also assigned in pathways related to mRNA surveillance, pyrimidine metabolism, and spliceosome. Figure $5 \mathrm{a}, \mathrm{b}$ show the detailed distribution of differentially methylated genes (DMGs) in pathways by Map-Man. Plentiful DMGs were involved in pathways of 'signaling' and hormone signaling. Moreover, low-P-related transcription factors, including ERF, $b H L H, W R K Y, N A C$, and MYB members, involved differences in methylation. In addition, the methylation levels of genes played roles in ion transport, lipid metabolism, and stress response were also disrupted (Figure 5a,b).

While investigating the change in methylation of transcription factors (TFs) under low-P stress, 570 TFs showed alterations in methylation in NN_LP vs NN_HP. The TFs consisted of 358 hypermethylated genes and 212 hypomethylated genes. A total of 363 and 212 of 575 TFs showed hyper- or hypomethylation in BG_LP vs BG_HP, respectively (Table S3). The two cultivars shared 90 common hypermethylated TFs and 29 hypomethylated TFs under low-P stress (Figure 5c). Previous studies reported the different tolerance to $\mathrm{P}$ of ' $\mathrm{NN}$ ' and ' $\mathrm{BG}$ ' indicated that ' $\mathrm{NN}$ ' is a low-P-tolerant accession and 'BG' is a low-P-sensitive accession. To explore the potential differentially methylated TFs yielding the difference of $\mathrm{P}$ tolerance in ' $\mathrm{NN}$ ' and 'BG,' we analyzed the noncommon methylation alterations among NN_LP vs NN_HP and BG_LP vs BG_HP. Interestingly, a GRAS transcription factor family member, SCARECROW-LIKE (SCL9), presented remarkable gene body hypermethylation in NN_LP vs NN_HP (Figure 5d) but was unaltered in 'BG' under low-P conditions (Figure S9). Moreover, the expression analysis showed that GmSCL9 mRNA abundance was repressed in NN_LP vs NN_HP but slightly induced in BG_LP vs BG_HP (Table S4). Arabidopsis SCL9 is homologous to gibberellin-insensitive (AtGAI) and repressor of ga1-3 (AtRGA), which act as negative regulators of GA signal transduction, and the inactivation of which largely modulate growth-promoting effect on primary roots [52]. Correspondingly, GmSCL9 was downregulated in 'NN' (low-P-tolerant) and non-significantly upregulated in 'BG' (low-P- sensitive) under low-P stress (Table S4). These results indicated that the difference in low-P-tolerance between ' $\mathrm{NN}^{\prime}$ ' and ' $\mathrm{BG}$ ' might be due to the differential expression of GmSCL9 in 'NN' and 'BG.' Figure S10 illustrates the six additional typical hypomethylation regions, including WRKY, which encode a homolog of AtWRKY6 (AT1G62300) in Arabidopsis and modulates Phosphate1 (Pho1) expression in response to low-P stress, ERF, bHLH, MYB, ARF, and NAC (Figure S10). 
a
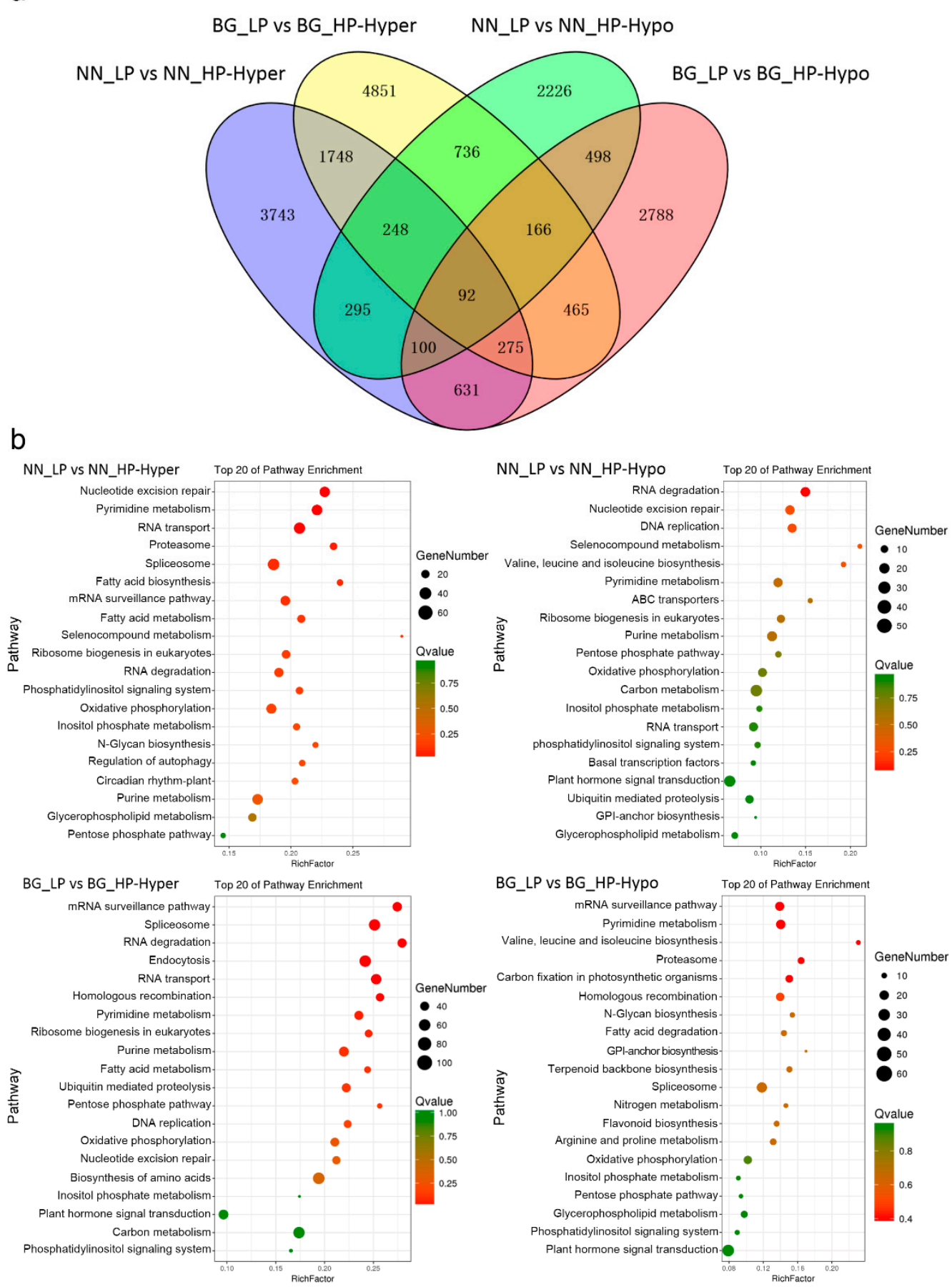

Figure 4. Differential methylome analysis under low-P stress. (a) Venn diagram of hyper/ hypomethylated genes among 'Nan-nong94-156' and 'Bogao' under low-P stress; (b) KEGG pathway enrichment of hypermethylated and hypomethylated genes in two cultivars under low-P conditions. The size of the circle represents gene numbers, and the color represents the q-value. NN_LP vs NN_HP, 'NN' low-P versus high-P; BG_LP vs BG_HP, 'BG' low-P versus high-P. 
a

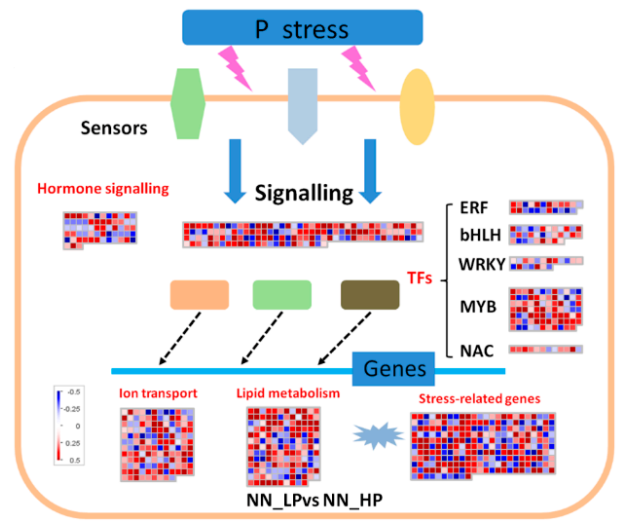

C

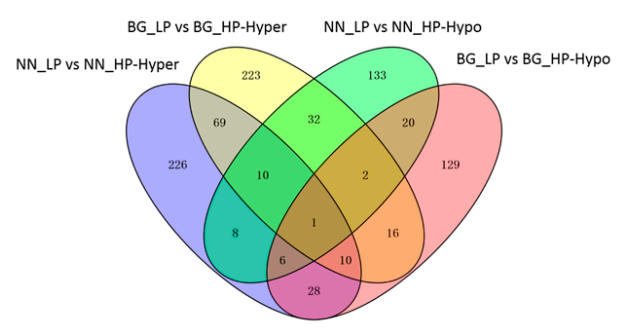

b

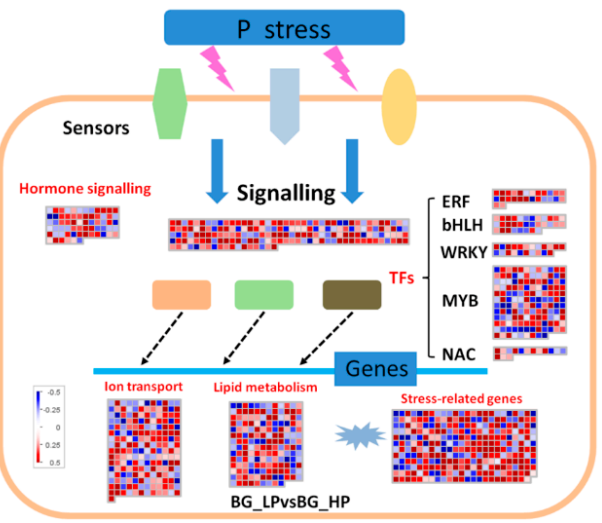

d

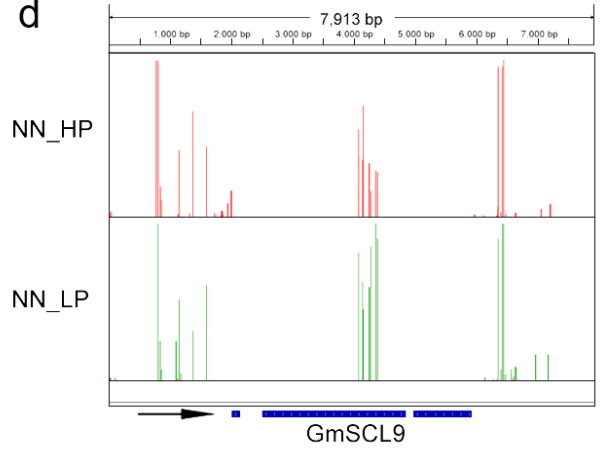

Figure 5. Assignment of differentially methylated genes among 'Nan-nong94-156' (a) and 'Bogao' (b) under low-P stress in Mapman bins. The red and blue squares indicate the hyper- and hypomethylated genes, respectively. Other different shaped graphics with different colors refer to some sensors and transcription factors (TFs) responding to low-P stress. (c) Venn map of differentially methylated transcriptional factors; (d) IGV software depicts the hypermethylation of GmSCL9 gene body region induced by low-P stress in 'Nan-nong94-156'. NN_LP vs NN_HP, 'NN' low-P versus high-P; BG_LP vs. BG_HP, ‘BG’ low-P versus high-P.

\subsection{Abundant TE Genes Are Hypomethylated in Response to Low-P Stress}

Transposable elements (TEs) are mobile DNA elements within the genome and their mobilization and silencing were reported to be associated with DNA methylation disruption $[7,24,53]$. The data showed that a higher number of TEs were associated with hypo-DMRs in both genotypes under low-P stress (Figure 6a). For NN_LP vs NN_HP, 123, 548, and 2172 hypermethylated and 179, 623, and 3079 hypomethylated TEs in $\mathrm{mCG}, \mathrm{mCHG}$, and $\mathrm{mCHH}$ sites were identified, respectively. For BG_LP vs BG_HP, 96, 480, and 2153 TEs were hypermethylated in $\mathrm{mCG}, \mathrm{mCHG}$, and $\mathrm{mCHH}$ contexts, respectively, whereas 182, 612, and 2578 TEs were hypomethylated (Figure 6a). As shown in the heat maps in Figure 6b, the methylation changes were further exhibited in differentially methylated TEs in each methylation context among NN_LP vs NN_HP and BG_LP vs BG_HP. The results suggested that abundant differentially methylated TEs showed demethylation at each methylation sequence context among NN_LP vs NN_HP and BG_LP vs BG_HP. Moreover, the NN_LP vs NN_HP combination presented much more hypomethylated TEs in the CHH context in contrast with BG_LP vs BG_HP. It was reported previously that methylation levels within TEs might dynamically regulate the expression of transposon genes and the near genes in the process of coping with stress [54]. Additionally, the demethylation effect on TEs in this study may be related to the regulation of transposons and genes involved in the response to low-P stress. 
a

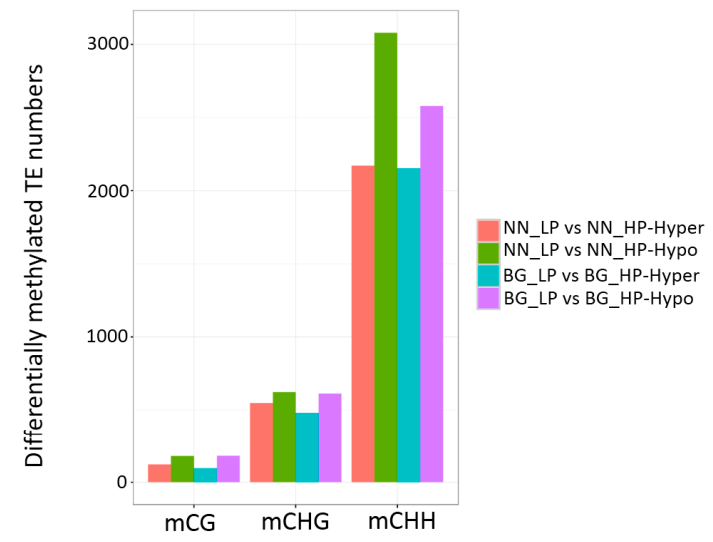

b

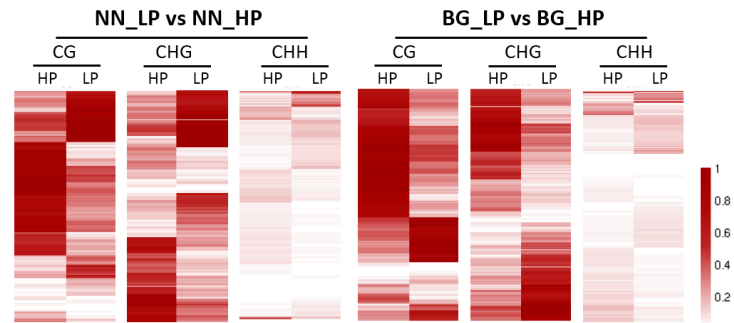

Figure 6. Differentially methylated TEs. (a) Numbers of differentially methylated TEs in 'Nan-nong94-156' or 'Bogao' under low-P stress; (b) heat maps of differentially methylated TEs. NN_LP vs NN_HP, ‘NN' low-P versus high-P; BG_LP vs BG_HP, ‘BG’ low-P versus high-P.

\subsection{Conjoint Analysis of Methylome and Transcriptome Alterations in Low-P Stress}

To explore the gene expression alterations accompanied by widespread methylation changes in response to low-P stress, RNA-seq analysis was performed with the same accessions grown either under HP or LP conditions. In total, 1002 and 1224 genes were differentially expressed in 'NN' and 'BG,' respectively (Table S4). In brief, 408 genes were upregulated and 594 genes were downregulated in NN_LP vs NN_HP, while 595 upregulated genes and 629 downregulated genes were present in BG_LP vs BG_HP (Figure 7a). These results suggested that more differentially expressed genes (DEGs) were identified in 'BG' than in ' $\mathrm{NN}^{\prime}$ and that there was a tendency for more downregulated genes in both ' $\mathrm{NN}$ ' and 'BG' upon low-P treatment. The hierarchical clustering analysis of genome-wide transcriptional alterations showed distinct differences in the way of the tolerant and sensitive accessions responding to the low-P stress (Figure $7 \mathrm{~b}$ ). To investigate the effect of methylation changes on transcriptional alterations, we identified low-P-induced DMGs associated with DEGs. Altogether, 65 hyper-DMGs overlapped with downregulated DEGs, and 17 hypo-DMGs overlapped with upregulated DEGs in NN_LP vs NN_HP. Nevertheless, 42 upregulated DEGs and 30 downregulated DEGs overlapped with hyper-DMGs and hypo-DMGs, respectively (Figure 7c). Similarly, in BG_LP vs BG_HP, 80 DEGs were downregulated with hypermethylation, and 43 DEGs were upregulated with hypomethylation. However, 79 upregulated DEGs and 45 downregulated DEGs overlapped with hyper-DMGs and hypo-DMGs, respectively (Figure 7d). 


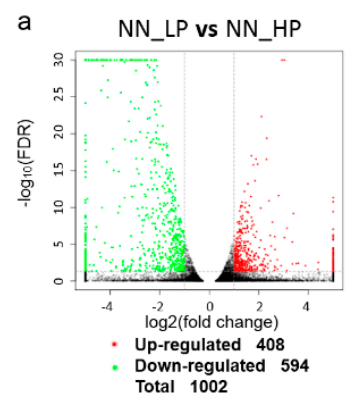

Dotal 1002
Totan

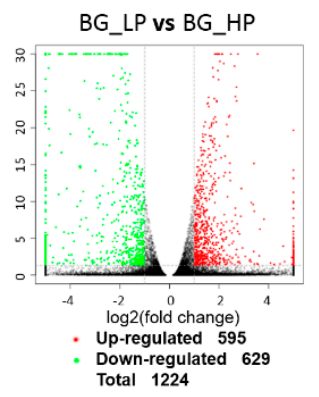

Total 1224
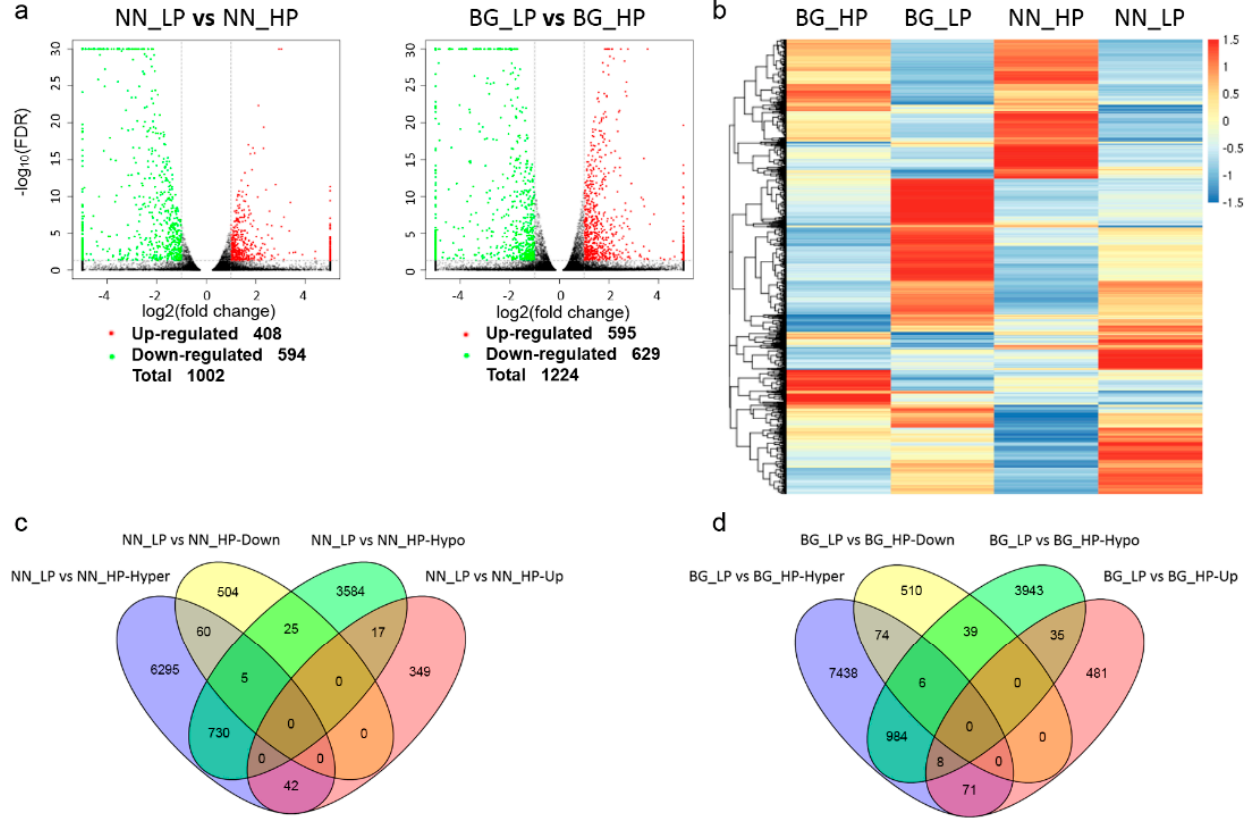

d

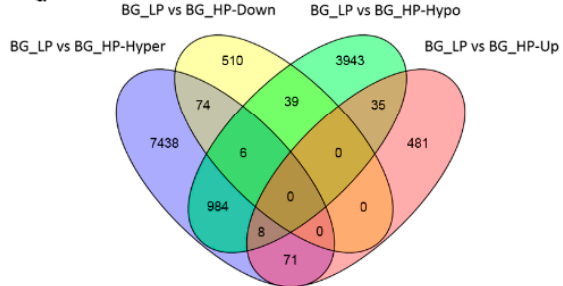

e
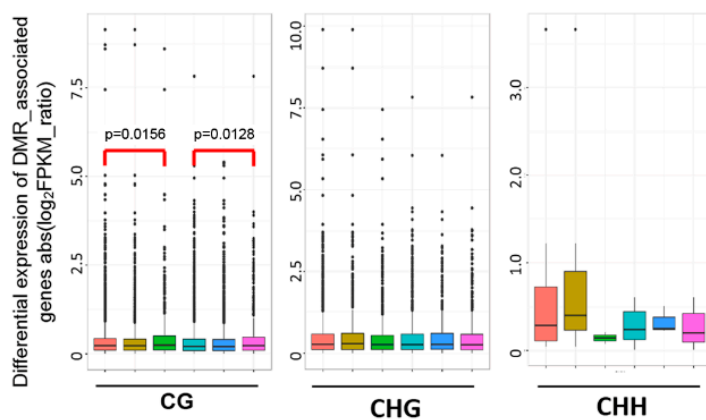

官 NN_LP vs NN_HP_All 自NN_LP vs NN_HP_Hyper NN_LP vs NN_HP_Hypo BG_LPvs BG_HP_All 官BG_LPvs BG_HP_Hyper 官BG_LP vs BG_HP_Hypo

Figure 7. The effect of methylation changes on transcriptional alterations. (a) Differentially expressed genes (DEGs) in 'Nan-nong94-156' or 'Bogao' in response to low-P stress. Each dot represents one gene. The red dots represent upregulated genes and the green dots represent downregulated genes. The black dots represent genes without differential expression. The $X$-axis is the $\log 2$ value of fold change and the Y-axis is the $\log 10$ value of false discovery rate (FDR); (b) heat maps of DEGs. NN_HP, 'Nan-nong94-156' under control conditions; NN_LP, ‘Nan-nong94-156' under low-P conditions; BG_HP, 'Bogao' under control conditions; BG_LP, 'Bogao' under low-P conditions. Venn diagram of DMGs (differentially methylated genes) and DEGs in NN_LP vs NN_HP (c) and BG_LP vs BG_HP (d); (e) differential expression levels of all genes (red box), hypermethylated genes (green box), and hypomethylated genes (blue box) among NN_LP vs NN_HP and BG_LP vs BG_HP in three sequence contexts (CG, CHG, and $\mathrm{CHH}$ ) are displayed as boxplots (boxes represent the quartiles; Wilcoxon $\mathrm{P}$ values are reported). NN_LP vs NN_HP, ‘NN' low-P versus high-P; BG_LP vs BG_HP, ‘BG’ low-P versus high-P.

A list of the low-P-induced and methylation-changed genes and their gene IDs, methylation levels, the corresponding associated expression patterns, and functional annotation is shown in Table S5. Furthermore, we compared the expression levels of all genes with hyper-DMGs or hypo-DMGs. As shown in Figure 7e, in both 'NN' and 'BG' under low-P stress, hypo-DMR genes showed significantly higher expression levels compared with all genes in the $\mathrm{mCG}$ context. In the mCHG context, both hyper-DMGs and hypo-DMR genes did not present significant differences in expression levels compared to all genes. Additionally, in the $\mathrm{mCHH}$ context, although the presence of slightly higher expression levels of hyper-DMR genes and the slightly lower expression levels of hypo-DMR genes 
were detected compared with all genes, there was no statistically significant difference with $p>0.05$ (Wilcoxon test) between hypomethylated or hypermethylated genes and all genes.

This finding indicates that there was no association between most of the transcript abundance varieties and methylation alterations. Altogether, these data indicate that DNA methylation is partially involved in the transcriptional changes of these genes. A portion of the differentially expressed genes is more a consequence of methylation-dependent alterations in transcriptional networks than a direct target of DNA methylation.

\subsection{Association Analyses of DNA Methylation and Small RNA Expression}

As increasing evidence has indicated that de novo DNA methylation is mediated by RNA-directed DNA methylation (RdDM) pathways, which are guided by small RNAs, we investigated the relationship between small RNA expression and DNA methylation $[8,55,56]$. The small RNA expression profiles of the same materials as methylome analysis were achieved by high-throughput deep sequencing. As RdDM is guided mainly by 24-nucleotide (nt) small interfering RNAs (siRNAs) and the 24-nt class was the most abundant group of small RNAs in soybean roots based on their length distribution (Figure S11), we focused on the 24-nt siRNA covered regions for subsequent investigations [57].

We compared the methylation levels between 24-nt siRNA covered regions and the regions without siRNA coverage in each methylation context. The results showed that the methylation level in all three methylation contexts in siRNA regions was significantly increased compared with the regions without siRNA (Figure S12). In addition, the 24-nt siRNA abundance in TE regions rises to a peak near transcriptional start sites and transcriptional end sites and decreases sharply when departing from these sites (Figure 8). In particular, the 24-nt siRNA abundance through all the TE regions in both 'BG' and ' $\mathrm{NN}$ ' in LP conditions was substantially lower than that in HP conditions, which was consistent with the $\mathrm{CHH}$ methylation pattern in the same region (Figures $2 \mathrm{~b}$ and 8 ). These results indicated that 24-nt siRNAs could be responsible for the reduction of the DNA methylation level, especially for the $\mathrm{CHH}$ sequence contexts (Figures $2 \mathrm{~b}$ and 8 ).

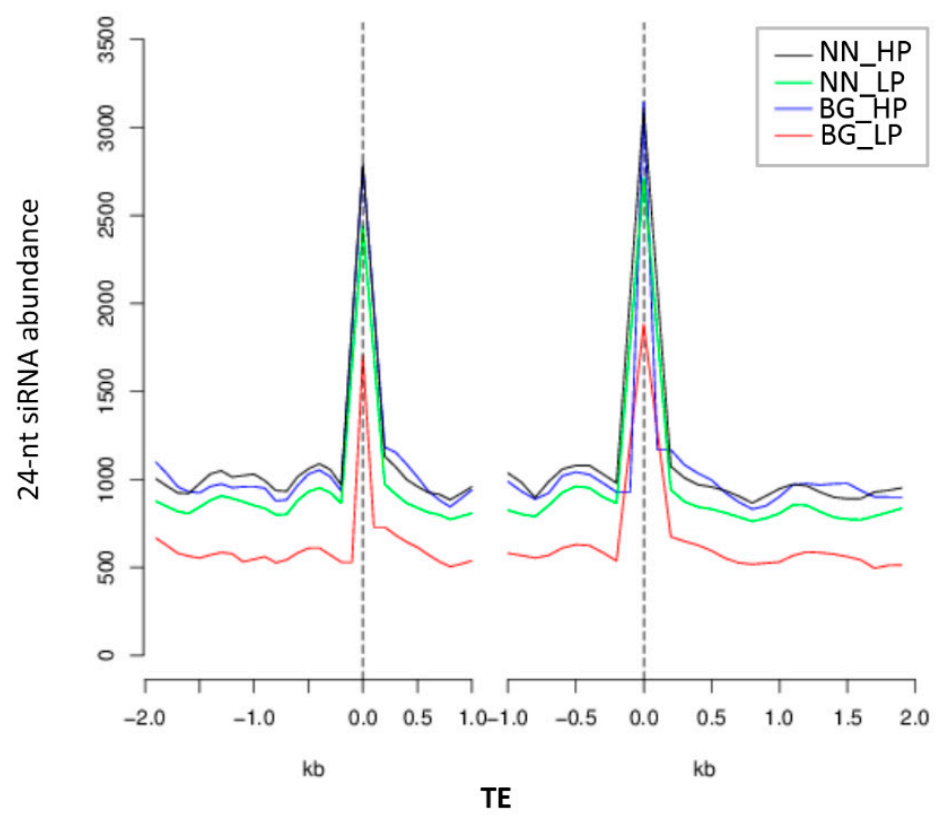

Figure 8. Number distribution of siRNAs in the TE and flanking 2-kb regions. NN_HP represents 'Nan-nong94-156' under control conditions; NN_LP represents 'Nan-nong94-156' under low-P conditions; BG_HP represents 'Bogao' under control conditions, and BG_LP represents 'Bogao' under low-P conditions. 


\section{Discussion}

DNA methylation has become one of the most heavily researched topics in plant functional genomics because of its important role in modulating plant plasticity in response to various stresses [47]. Low-P stress is one of the most important abiotic stresses in soybean. Using the whole-genome bisulfite sequencing approach, we evaluated changes in methylation genome-wide when suffering low-P stress in two soybean varieties with different levels of low-P tolerance. Our results revealed that DNA methylation levels were slightly higher under low-P stress and low-P-induced methylome changes partially related to the changes in gene expression and siRNA abundance. The available data sets of our study could be applied to select potent epigenetic regions as probable targets for genetic manipulation strategies for crop improvement to engineer tolerance against abiotic stresses.

Whole-genome bisulfite sequencing (WGBS) in single-base resolution provided an overall view of methylation patterns of the two soybean accessions, presenting an average DNA methylation level of $67.54 \% \mathrm{mCG}$ (mCG/CG), 44.57\% mCHG (mCHG/CHG), and 3.79\% mCHH (mCHH/CHH), respectively. Clearly, soybean methylation levels are moderate among diverse reported species. Considering the moderate genome size $(978 \mathrm{Mb})$ of soybean, our results provide evidence to confirm the positive correlation between methylation levels and genome sizes [58,59]. It was reported previously that the representative hypermethylation regions were centromeres and peri-centromeric areas. Moreover, the negative correlation between methylation levels and gene number was revealed by several previous studies $[21,59,60]$. Consistently, our results also demonstrated the positive correlation of CG, CHG, and $\mathrm{CHH}$ methylation levels with TE density and the negative correlation with gene number, which suggests the maintenance of genome stability as a primary function of DNA methylation.

DNA methylation has been reported to repress gene expression [61]. However, in recent years, numerous genomic methylation investigations have shown that the correlation between DNA methylation and transcription is slightly different than initially recognized. For example, the recent rice methylome analysis showed that gene body methylation usually presented a positive correlation with gene expression [62]. An investigation in Arabidopsis revealed that DNA methylation was only marginally responsible for gene expression [63]. Additionally, a more recent study in apple indicated that there is no apparent relationship between promoter methylation and gene expression [64]. However, in our study, the results showed that non-expressed genes possessed relatively high methylation levels in each methylation sequence context, and the CG and CHG methylation levels exhibited an inverse correlation with expression throughout broad regions of the gene. Surprisingly, although the $\mathrm{CHH}$ methylation level was negatively correlated with expression in the gene body, the $\mathrm{CHH}$ methylation level of highly expressed genes was higher than expressed genes with low or moderate expression levels in promoter and downstream regions (Figure 3a).

Furthermore, genes with the highest methylation levels showed the lowest expression levels in both promoter and gene body regions, while no significant difference was found among the different methylation-level quintiles. In short, all the results indicated a more complex regulatory correlation between DNA methylation and gene expression which appears to depend on the type, region, and extent of methylation, as well as species.

Low-P stress is one of the most important abiotic stresses, while related DNA methylation pattern studies only focused on a few model plants, such as Arabidopsis and rice [39-41]. Our study is the first to report the DNA methylation alterations in response to low-P stress in legume model plant soybean. In Arabidopsis, P starvation could lead to a series of changes in root architecture, including the great increase in density and length of lateral roots and root hairs as well as inhibited growth of primary roots. In contrast, the length of the primary root in soybean is also induced by low-P stress. Based on the difference in phenotypic changes in response to low-phosphorus stress, we hypothesized that there is also a difference in methylation changes between soybean and Arabidopsis. Therefore, we evaluated genome-wide methylation changes in response to low $\mathrm{P}$ using two representative soybean varieties with low-P tolerance and low-P sensitivity. It is noteworthy that only one type of accession was used in previous methylome studies of $\mathrm{P}$ starvation response in Arabidopsis and rice. Our results revealed 
that both the low-P-tolerant accession ' $\mathrm{NN}^{\prime}$ and the low-P-sensitive accession 'BG' exhibit similar dynamic changes in the DNA methylation pattern exposed to low-P availability, which was slightly elevated methylation levels in each methylation sequence context. In contrast, the methylation levels increased by up to 1.5-fold in 7-day-old LP seedlings in comparison with age-matched HP seedlings in Arabidopsis [40]. The difference in changes in methylation levels could be a possible explanation for phenotypic differences in the low-P response between Arabidopsis and soybean. Furthermore, interestingly, ' $\mathrm{NN}$ ' presented a smaller amount of low-P-induced differentially methylated regions compared with 'BG' in each methylation context. The relatively milder methylation changes in ' $\mathrm{NN}^{\prime}$ than in 'BG' may better maintain genomic stability when suffering low-P conditions. In addition, ' $\mathrm{NN}$ ' and 'BG' only shared approximately one-third and one-fifth of the hypermethylated genes and hypomethylated genes, respectively. The more noncommon methylation alterations may contribute to the significantly differential tolerance to low-P stress between 'NN' and 'BG.'

DNA methylation alterations under various stress conditions are often associated with the regulation of gene expression. Accordingly, whether and how DNA methylation is correlated with gene expression under $\mathrm{P}$ deficiency is presented and discussed in our study. The results indicate that only a small portion of the low-P stress-induced regions of differential methylation overlapped with genes of differential expression. Most alterations in gene expression were not associated with the corresponding methylation changes. This finding is consistent with the previous observation that a minor fraction of DMRs was correlated with altered gene expression in maize under nutrient deficiencies [65]. This phenomenon could be partially explained by the fact that changes in methylation in some regions do not affect gene expression. It can also be explained that the change of a large number of gene expression is not directly regulated by methylation, and methylation may indirectly affect the transcription network through directly regulating the expression of genes located upstream of the network. This indirect regulation can be regulated by a variety of mechanisms. For example, chilling stress altered the DNA methylation status of RIPENING INHIBITOR (RIN), NONRIPENING, and COLORLESS NONRIPENING, which encode transcription factors necessary for ripening, and decreased the transcript levels of these genes and their downstream genes in tomato [66]. Similarly, the methylation and expression levels of the transcription factor gene families $M Y B, b-Z I P$, and $A P 2 / D R E B$ presented significant correlations in soybean during salinity stress [67]. Our results also suggested that many transcription factor genes presented low-P-associated methylation alterations involving members of the NAC, WRKY, ERF, ARF, and $b H L H$ classes. For instance, a homolog of auxin response factor 19 (ARF19), which positively regulates PHOSPHATE STARVATION RESPONSE 1, a central regulatory system of P-responsive genes in Arabidopsis roots, was observed to be changed of DNA methylation status and transcripts in our study [68]. It is understandable that plants tend to control TFs compared with regulating structural genes to further regulate biological pathways, which seems to be a more energy-efficient means of coping with environmental stress.

One striking finding of our analysis is that differentially methylated regions are abundantly present in transposable elements, indicating that DNA methylation related to low P availability is necessary for maintaining genome integrity. Furthermore, abundant differentially methylated TEs showed demethylation at each methylation sequence context. In agreement with this finding, it has been recently reported that most TEs were hypomethylated in $\mathrm{Zn}$ maize roots [69]. The level of DNA methylation is important for controlling TE activity, and the changed activity of TEs might significantly affect the expression of nearby genes to enhance adaptational processes to abiotic stress, such as $P, N$, and Zn deficiency $[65,70,71]$. Accordingly, the dynamic DNA demethylation within TEs observed in this investigation may regulate the transcriptional changes of transposons and proximal genes responding to low-P stress. In addition, the low-P-induced demethylation in TEs, especially in the $\mathrm{CHH}$ methylation context, is accompanied by a tremendous reduction in the amount of siRNAs, which was consistent with previous observations of the abundance of siRNAs presented to be positively correlated with DNA methylation in rice and maize subjected to salt stress and Zn deficiency, respectively [69,72]. Furthermore, the methylation levels at each methylation sequence context were significantly higher 
in siRNA mapping regions than the regions without siRNA mapping. These results indicated that a cross-talk existed between the different methylation pathways, such as the RdDM pathway, to maintain the methylation level.

\section{Materials and Methods}

\subsection{Plant Materials and Treatment}

The low-P tolerance genotype 'Nannong 94156' (NN) and deficient P-sensitive genotype 'Bogao' (BG) were grown in hydroponic culture, as described in our previous report [73]. All plants were grown in an artificial climate chamber with a $10 \mathrm{~h} / 14 \mathrm{~h}$ (day/night) photoperiod and a temperature cycle of $28^{\circ} \mathrm{C} / 20^{\circ} \mathrm{C}$ (day/night). First, the surface-sterilized seeds were germinated in sterile vermiculite. When the two cotyledons were fully expanded, soybean seedlings were transferred into modified $1 / 2$ Hoagland's nutrient solution for $3 \mathrm{~d}$. Then, half of the seedlings were transferred to modified Hoagland's nutrient solution at a one-half strength with lacking $\mathrm{P}\left(5 \mu \mathrm{M} \mathrm{P}, \mathrm{KH}_{2} \mathrm{PO}_{4}\right.$, low-P, LP), and the other half were transferred into modified Hoagland's nutrient solution at a one-half strength supplemented with $500 \mu \mathrm{M} \mathrm{P}\left(\mathrm{KH}_{2} \mathrm{PO}_{4}\right.$, high-P, $\left.\mathrm{HP}\right)$ as controls. These treatments continued for 7 days and the nutrient solution was exchanged every three days. The samples in our study were designated based on the treatments as follows: NN_HP, 'Nan-nong94-156' under control conditions; NN_LP, 'Nannong94-156' under low-P stress; BG_HP, 'Bogao' under control conditions; BG_LP, 'Bogao' under low-P stress. Finally, root tissues of nine representative plants from each treatment were harvested and stored at $-70{ }^{\circ} \mathrm{C}$ for further use.

\subsection{Bisulfite Sequencing Library Construction}

The root tissues of the nine plants of each treatment were pooled to one biological replicate. Genomic DNA was extracted from roots using a modified CTAB method [74]. DNA concentration and integrity were detected by a NanoDrop spectrophotometer and Agarose Gel Electrophoresis, respectively. Briefly, 1 ug of genomic DNA plus unmethylated $\lambda$ DNA was interrupted into 100-300 bp fragments and purified using a Sonication and MiniElute PCR Purification Kit (QIAGEN, Hilden, Germany). The end-repaired genomic fragments were ligated with a single " $\mathrm{A}$ " nucleotide in the 3 " end and then with the methylated sequencing adapters. Then, these fragments were bisulfite converted with the Methylation-Gold Kit (ZYMO, Orange, CA, USA). Finally, the processed DNA fragments were subjected to PCR amplification and double-end sequencing using the Illumina HiSeqTM 4000 platform.

\subsection{Read Mapping and Methylation Level Analysis}

To obtain high-quality clean reads, raw reads were filtered based on the following rules: (i) removing reads involving more than $10 \%$ of unknown nucleotides $(\mathrm{N})$ and (ii) removing low-quality reads containing more than $40 \%$ of low-quality $(\mathrm{Q}$-value $\leq 20)$ bases. The obtained clean reads were mapped to the soybean genome (Glycine max Wm82.a2.v1) using BSMAP software (version: 2.90, Baylor College of Medicine, Houston, TX, USA) by default [75]. Sequencing reads produced during this study have been deposited in the National Center for Biotechnology Information Sequence Read Archive (NCBI SRA accession numbers SRP233333). Then, the methylation level according to the methylated cytosine percentage in the whole genome and in different regions of the genome was calculated using a custom Perl script. Additionally, the methylation profile at flanking 2-kb regions and the gene body (or transposable elements) was plotted according to the average methylation levels of each 100-bp interval to evaluate different methylation patterns in different genomic regions.

\subsection{Differentially Methylated Regions (DMRs) Analysis}

Differentially methylated regions (DMRs) were identified based on the following criteria: (1) at least five methylated cytosines are present in more than one sample; (2) each methylated cytosine is covered by at least four reads; (3) region length is between $40 \mathrm{bp}$ and $10 \mathrm{~kb}$; (4) the distance between adjacent 
methylated is less than $200 \mathrm{bp}$; (5) the change of the average methylation level is more than two-fold; (6) Pearson's chi-square test $\left(\chi^{2}\right)$ value yields a $p$-value $\leq 0.05$. To analyze the functional enrichment of genes affected by DMRs, gene ontology (GO) enrichment analysis (http://www.geneontology.org/) and KEGG pathway enrichment analysis (http://www.kegg.jp/kegg/) were conducted for DMR-related genes by the hypergeometric test with a corrected $p$-value $\leq 0.05$.

\subsection{RNA Sequencing and Transcriptome Profiling Analysis}

For transcriptome analysis, the root tissues from the nine plants used for methylome analysis were pooled into three samples of three plants each to form three independent biological replicates for each treatment [76]. Total RNA was extracted using Trizol (Life Technologies Inc., Gaithersburg, MD, USA). The RNA quality was tested using an Agilent 2100 Bioanalyzer (Santa Clara, CA, USA), and the quantity was tested using a NanoDrop spectrophotometer (Waltham, MA, USA). The extracted total RNA was purified using Oligo (dT) magnetic beads. Then, the mRNA was fragmented with fragmentation buffer. The short mRNA fragments were reverse transcribed into cDNA by random primers, DNA polymerase I, RNase H, dNTPs, and buffer. Then, the cDNA fragments were purified with a QiaQuick PCR extraction kit. After end-repair, end-addition of A, and ligation to Illumina sequencing adapters, the size of the ligation products was selected by agarose gel electrophoresis followed by PCR amplification and sequencing using the Illumina HiSeqTM 4000 platform. Sequencing raw data was then filtered to remove sequencing adapters and low-quality reads. The clean reads were aligned to the soybean reference genome (Wm82. a2) by TopHat v2.0.3.12 [77]. Sequencing reads generated by this study are available from the NCBI Sequence Read Archive (SRA) Database (accession numbers SRP233239). The calculation of estimated expression abundance was conducted using the Cufflinks package [78]. The FPKM (fragments per kilobase of transcript per million mapped reads) was calculated based on their length and read count, which was used to estimate the transcript abundance of each gene. The edgeR package was applied for differential gene expression analysis (http://www.r-project.org/). The differentially expressed genes were identified according to a fold change $\geq 2$ and a false discovery rate (FDR) $<0.05$ and were further analyzed by cluster analysis using the "heatmap.2" function in the gplots package of $\mathrm{R}$ (https://www.r-project.org/).

\subsection{Small RNA Sequencing and Data Analysis}

Three biological replicates were used for small RNA sequencing. The RNA molecules measuring 18-30 nt were first enriched by polyacrylamide gel electrophoresis (PAGE). The purified small RNA was then ligated to sequencing adapters and performed PCR amplification. The constructed cDNA library was finally sequenced by the Illumina HiSeq TM 4000 platform. After sequencing, the raw data obtained were filtered, including the removal of sequencing adapters and low-quality reads. In addition, reads with identical sequences, including ribosomal RNA, tRNA, small nucleolar RNA, and small nuclear RNA, were removed from the raw data. All of the clean small RNA sequences were mapped to the soybean genome by the SOAP 2.0 program [79]. Only the unique sequences were subjected to subsequent analysis. Sequencing reads generated by this study have been deposited in NCBI's database of SRA (accession numbers SRP233151).

\section{Conclusions}

Taken together, the results of this work indicate that DNA methylation alterations could affect gene expression in both direct and indirect ways in response to low-P stress in soybean. The indirect alterations in gene expression may be caused by DNA methylation regulating gene expression upstream of the transcription network. Low-P-induced methylation changes were enriched in TEs, and changes in $\mathrm{CHH}$ methylation levels in TE regions were accompanied by changes in the amount of siRNA, which indicated that siRNAs could play an important role in regulating TE activity by guiding $\mathrm{CHH}$ methylation in TE regions. Our genome-wide perspective revealed unique aspects of methylome 
changes induced by low P in soybean. These data would be beneficial for studying the epigenetic regulation of abiotic stress responses in plants.

Supplementary Materials: The following are available online at http://www.mdpi.com/1422-0067/21/18/6817/s1.

Author Contributions: D.Z. and S.C. conceived and designed the experiments. S.C., X.Z., K.Y. and L.L. performed the experiments. S.C., C.S., X.L., J.Z. and Y.J. performed the data analyses. S.C. and D.Z. wrote the manuscript. All authors have read and agreed to the published version of the manuscript.

Funding: This research was funded by the key scientific and technological project of the Henan Province (192102110023), the Henan agricultural university science and technology innovation fund (KJCX2019C02), the Ministry of Science and Technology of China (2016YFD0100500), and the China Postdoctoral Science Foundation (2017M612400).

Conflicts of Interest: The authors declare no conflict of interest.

\section{Abbreviations}

$\begin{array}{ll}\text { P } & \text { Phosphorus } \\ \text { TE } & \text { Transposable element } \\ \text { RdDM } & \text { RNA-directed DNA methylation } \\ \text { NN } & \text { Nannong 94-156 } \\ \text { BG } & \text { Bogao } \\ \text { LP } & \text { Low-P } \\ \text { HP } & \text { High-P } \\ \text { DMRs } & \text { Differentially methylated regions } \\ \text { GO } & \text { Gene Ontology } \\ \text { KEGG } & \text { Kyoto Encyclopedia of Genes and Genomes } \\ \text { DMGs } & \text { Differentially methylated genes } \\ \text { TFs } & \text { Transcription factors } \\ \text { DEGs } & \text { Differentially expressed genes } \\ \text { SiRNAs } & \text { Small interfering RNAs }\end{array}$

\section{References}

1. Hirayama, T.; Shinozaki, K. Research on plant abiotic stress responses in the post-genome era: Past, present and future. Plant J. 2010, 61, 1041-1052. [CrossRef] [PubMed]

2. Chiou, T.J.; Lin, S.I. Signaling network in sensing phosphate availability in plants. Annu. Rev. Plant Biol. 2011, 62, 185-206. [CrossRef] [PubMed]

3. Mirouze, M.; Paszkowski, J. Epigenetic contribution to stress adaptation in plants. Curr. Opin. Plant Biol. 2011, 14, 267-274. [CrossRef] [PubMed]

4. $\quad$ Feng, S.; Cokus, S.J.; Zhang, X.; Chen, P.Y.; Bostick, M.; Goll, M.G.; Hetzel, J.; Jain, J.; Strauss, S.H.; Halpern, M.E.; et al. Conservation and divergence of methylation patterning in plants and animals. Proc. Natl. Acad. Sci. USA 2010, 107, 8689-8694. [CrossRef]

5. Zemach, A.; McDaniel, I.E.; Silva, P.; Zilberman, D. Genome-wide evolutionary analysis of eukaryotic DNA methylation. Science 2010, 328, 916-919. [CrossRef]

6. Kou, H.P.; Li, Y.; Song, X.X.; Ou, X.F.; Xing, S.C.; Ma, J.; Von Wettstein, D.; Liu, B. Heritable alteration in DNA methylation induced by nitrogen-deficiency stress accompanies enhanced tolerance by progenies to the stress in rice (Oryza sativa L.). J. Plant Physiol. 2011, 168, 1685-1693. [CrossRef]

7. Sahu, P.P.; Pandey, G.; Sharma, N.; Puranik, S.; Muthamilarasan, M.; Prasad, M. Epigenetic mechanisms of plant stress responses and adaptation. Plant Cell Rep. 2013, 32, 1151-1159. [CrossRef] [PubMed]

8. Law, J.A.; Jacobsen, S.E. Establishing, maintaining and modifying DNA methylation patterns in plants and animals. Nat. Rev. Genet. 2010, 11, 204-220. [CrossRef]

9. Stroud, H.; Greenberg, M.V.; Feng, S.; Bernatavichute, Y.V.; Jacobsen, S.E. Comprehensive analysis of silencing mutants reveals complex regulation of the Arabidopsis methylome. Cell 2013, 152, 352-364. [CrossRef]

10. Niederhuth, C.E.; Schmitz, R.J. Covering your bases: Inheritance of DNA methylation in plant genomes. Mol. Plant 2014, 7, 472-480. [CrossRef] 
11. Vongs, A.; Kakutani, T.; Martienssen, R.A.; Richards, E.J. Arabidopsis thaliana DNA methylation mutants. Science 1993, 260, 1926-1928. [CrossRef] [PubMed]

12. Cao, X.; Jacobsen, S.E. Locus-specific control of asymmetric and CpNpG methylation by the DRM and CMT3 methyltransferase genes. Proc. Natl. Acad. Sci. USA 2002, 99 (Suppl. 4), 16491-16498. [CrossRef] [PubMed]

13. Kankel, M.W.; Ramsey, D.E.; Stokes, T.L.; Flowers, S.K.; Haag, J.R.; Jeddeloh, J.A.; Riddle, N.C.; Verbsky, M.L.; Richards, E.J. Arabidopsis MET1 cytosine methyltransferase mutants. Genetics 2003, 163, 1109-1122.

14. Penterman, J.; Zilberman, D.; Huh, J.H.; Ballinger, T.; Henikoff, S.; Fischer, R.L. DNA demethylation in the Arabidopsis genome. Proc. Natl. Acad. Sci. USA 2007, 104, 6752-6757. [CrossRef]

15. Ortega-Galisteo, A.P.; Morales-Ruiz, T.; Ariza, R.R.; Roldan-Arjona, T. Arabidopsis DEMETER-LIKE proteins DML2 and DML3 are required for appropriate distribution of DNA methylation marks. Plant Mol. Biol. 2008, 67, 671-681. [CrossRef]

16. Chinnusamy, V.; Zhu, J.K. Epigenetic regulation of stress responses in plants. Curr. Opin. Plant Biol. 2009, 12, 133-139. [CrossRef]

17. Wang, W.S.; Pan, Y.J.; Zhao, X.Q.; Dwivedi, D.; Zhu, L.H.; Ali, J.; Fu, B.Y.; Li, Z.K. Drought-induced site-specific DNA methylation and its association with drought tolerance in rice (Oryza sativa L.). J. Exp. Bot. 2011, 62, 1951-1960. [CrossRef]

18. Karan, R.; DeLeon, T.; Biradar, H.; Subudhi, P.K. Salt stress induced variation in DNA methylation pattern and its influence on gene expression in contrasting rice genotypes. PLoS ONE 2012, 7, e40203. [CrossRef] [PubMed]

19. Chen, X.; Zhou, D.X. Rice epigenomics and epigenetics: Challenges and opportunities. Curr. Opin. Plant Biol. 2013, 16, 164-169. [CrossRef]

20. Cokus, S.J.; Feng, S.; Zhang, X.; Chen, Z.; Merriman, B.; Haudenschild, C.D.; Pradhan, S.; Nelson, S.F.; Pellegrini, M.; Jacobsen, S.E. Shotgun bisulphite sequencing of the Arabidopsis genome reveals DNA methylation patterning. Nature 2008, 452, 215-219. [CrossRef]

21. Lister, R.; O’Malley, R.C.; Tonti-Filippini, J.; Gregory, B.D.; Berry, C.C.; Millar, A.H.; Ecker, J.R. Highly integrated single-base resolution maps of the epigenome in Arabidopsis. Cell 2008, 133, 523-536. [CrossRef] [PubMed]

22. Liang, D.; Zhang, Z.; Wu, H.; Huang, C.; Shuai, P.; Ye, C.Y.; Tang, S.; Wang, Y.; Yang, L.; Wang, J.; et al. Single-base-resolution methylomes of Populus trichocarpa reveal the association between DNA methylation and drought stress. BMC Genet. 2014, 15 (Suppl. 1), S9. [CrossRef] [PubMed]

23. Brautigam, K.; Vining, K.J.; Lafon-Placette, C.; Fossdal, C.G.; Mirouze, M.; Marcos, J.G.; Fluch, S.; Fraga, M.F.; Guevara, M.A.; Abarca, D.; et al. Epigenetic regulation of adaptive responses of forest tree species to the environment. Ecol. Evol. 2013, 3, 399-415. [CrossRef]

24. Hashida, S.N.; Uchiyama, T.; Martin, C.; Kishima, Y.; Sano, Y.; Mikami, T. The temperature-dependent change in methylation of the Antirrhinum transposon Tam3 is controlled by the activity of its transposase. Plant Cell 2006, 18, 104-118. [CrossRef]

25. Qian, Y.; Cheng, X.; Liu, Y.; Jiang, H.; Zhu, S.; Cheng, B. Reactivation of a silenced minimal Mutator transposable element system following low-energy nitrogen ion implantation in maize. Plant Cell Rep. 2010, 29, 1365-1376. [CrossRef]

26. Lynch, J. Root Architecture and Plant Productivity. Plant Physiol. 1995, 109, 7-13. [CrossRef] [PubMed]

27. Raghothama, K.G. Phosphate Acquisition. Annu. Rev. Plant Physiol. Plant Mol. Biol. 1999, 50, 665-693. [CrossRef]

28. Vance, C.P. Symbiotic nitrogen fixation and phosphorus acquisition. Plant nutrition in a world of declining renewable resources. Plant Physiol. 2001, 127, 390-397. [CrossRef]

29. Poirier, Y.; Bucher, M. Phosphate transport and homeostasis in Arabidopsis. Arab. Book 2002, 1, e0024. [CrossRef] [PubMed]

30. Rouached, H.; Arpat, A.B.; Poirier, Y. Regulation of phosphate starvation responses in plants: Signaling players and cross-talks. Mol. Plant 2010, 3, 288-299. [CrossRef]

31. Ticconi, C.A.; Lucero, R.D.; Sakhonwasee, S.; Adamson, A.W.; Creff, A.; Nussaume, L.; Desnos, T.; Abel, S. ER-resident proteins PDR2 and LPR1 mediate the developmental response of root meristems to phosphate availability. Proc. Natl. Acad. Sci. USA 2009, 106, 14174-14179. [CrossRef] [PubMed] 
32. Rubio, V.; Linhares, F.; Solano, R.; Martin, A.C.; Iglesias, J.; Leyva, A.; Paz-Ares, J. A conserved MYB transcription factor involved in phosphate starvation signaling both in vascular plants and in unicellular algae. Genes Dev. 2001, 15, 2122-2133. [CrossRef] [PubMed]

33. Wang, C.; Ying, S.; Huang, H.; Li, K.; Wu, P.; Shou, H. Involvement of OsSPX1 in phosphate homeostasis in rice. Plant J. 2009, 57, 895-904. [CrossRef]

34. Kant, S.; Peng, M.; Rothstein, S.J. Genetic regulation by NLA and microRNA827 for maintaining nitrate-dependent phosphate homeostasis in arabidopsis. PLoS Genet. 2011, 7, e1002021. [CrossRef] [PubMed]

35. Secco, D.; Wang, C.; Arpat, B.A.; Wang, Z.; Poirier, Y.; Tyerman, S.D.; Wu, P.; Shou, H.; Whelan, J. The emerging importance of the SPX domain-containing proteins in phosphate homeostasis. New Phytol. 2012, 193, 842-851. [CrossRef] [PubMed]

36. Secco, D.; Jabnoune, M.; Walker, H.; Shou, H.; Wu, P.; Poirier, Y.; Whelan, J. Spatio-temporal transcript profiling of rice roots and shoots in response to phosphate starvation and recovery. Plant Cell 2013, 25, 4285-4304. [CrossRef]

37. Puga, M.I.; Mateos, I.; Charukesi, R.; Wang, Z.; Franco-Zorrilla, J.M.; de Lorenzo, L.; Irigoyen, M.L.; Masiero, S.; Bustos, R.; Rodriguez, J.; et al. SPX1 is a phosphate-dependent inhibitor of Phosphate Starvation Response 1 in Arabidopsis. Proc. Natl. Acad. Sci. USA 2014, 111, 14947-14952. [CrossRef]

38. Wang, Z.; Ruan, W.; Shi, J.; Zhang, L.; Xiang, D.; Yang, C.; Li, C.; Wu, Z.; Liu, Y.; Yu, Y.; et al. Rice SPX1 and SPX2 inhibit phosphate starvation responses through interacting with PHR2 in a phosphate-dependent manner. Proc. Natl. Acad. Sci. USA 2014, 111, 14953-14958. [CrossRef]

39. Secco, D.; Wang, C.; Shou, H.; Schultz, M.D.; Chiarenza, S.; Nussaume, L.; Ecker, J.R.; Whelan, J.; Lister, R. Stress induced gene expression drives transient DNA methylation changes at adjacent repetitive elements. Elife 2015, 4, e09343. [CrossRef]

40. Yong-Villalobos, L.; Gonzalez-Morales, S.I.; Wrobel, K.; Gutierrez-Alanis, D.; Cervantes-Perez, S.A.; Hayano-Kanashiro, C.; Oropeza-Aburto, A.; Cruz-Ramirez, A.; Martinez, O.; Herrera-Estrella, L. Methylome analysis reveals an important role for epigenetic changes in the regulation of the Arabidopsis response to phosphate starvation. Proc. Natl. Acad. Sci. USA 2015, 112, E7293-E7302. [CrossRef]

41. Yong-Villalobos, L.; Cervantes-Perez, S.A.; Gutierrez-Alanis, D.; Gonzales-Morales, S.; Martinez, O.; Herrera-Estrella, L. Phosphate starvation induces DNA methylation in the vicinity of cis-acting elements known to regulate the expression of phosphate-responsive genes. Plant Signal Behav. 2016, 11, e1173300. [CrossRef]

42. Li, X.; Chang, W.; Zhang, C. Advances of soybean (Glycine max L.) phosphorus nutrition and high P-eicient germplasms screen-ing in China. Soybean Sci. 2011, 30, 322-327.

43. Olivera, M.; Tejera, N.; Iribarne, C.; Ocana, A.; Lluch, C. Growth, nitrogen fixation and ammonium assimilation in common bean (Phaseolus vulgaris): Effect of phosphorus. Physiol. Plant. 2004, 121, 498-505. [CrossRef]

44. Zhang, D.; Liu, C.; Cheng, H.; Kan, G.; Cui, S.; Meng, Q.; Gai, J.; Yu, D. Quantitative trait loci associated with soybean tolerance to low phosphorus stress based on flower and pod abscission. Plant Breeding 2010, 129, 243-249. [CrossRef]

45. Zhang, D.; Song, H.; Cheng, H.; Hao, D.; Wang, H.; Kan, G.; Jin, H.; Yu, D. The acid phosphatase-encoding gene GmACP1 contributes to soybean tolerance to low-phosphorus stress. PLoS Genet. 2014, 10, e1004061. [CrossRef]

46. Elser, J.J. Phosphorus: A limiting nutrient for humanity? Curr. Opin. Biotechnol. 2012, 23, 833-838. [CrossRef]

47. Song, Q.X.; Lu, X.; Li, Q.T.; Chen, H.; Hu, X.Y.; Ma, B.; Zhang, W.K.; Chen, S.Y.; Zhang, J.S. Genome-wide analysis of DNA methylation in soybean. Mol. Plant. 2013, 6, 1961-1974. [CrossRef]

48. Hsieh, T.F.; Ibarra, C.A.; Silva, P.; Zemach, A.; Eshed-Williams, L.; Fischer, R.L.; Zilberman, D. Genome-wide demethylation of Arabidopsis endosperm. Science 2009, 324, 1451-1454. [CrossRef] [PubMed]

49. Zemach, A.; Kim, M.Y.; Silva, P.; Rodrigues, J.A.; Dotson, B.; Brooks, M.D.; Zilberman, D. Local DNA hypomethylation activates genes in rice endosperm. Proc. Natl. Acad. Sci. USA 2010, 107, 18729-18734. [CrossRef]

50. Lu, X.; Wang, W.; Ren, W.; Chai, Z.; Guo, W.; Chen, R.; Wang, L.; Zhao, J.; Lang, Z.; Fan, Y.; et al. Genome-Wide Epigenetic Regulation of Gene Transcription in Maize Seeds. PLoS ONE 2015, 10, e0139582. [CrossRef] 
51. Wang, H.; Beyene, G.; Zhai, J.; Feng, S.; Fahlgren, N.; Taylor, N.J.; Bart, R.; Carrington, J.C.; Jacobsen, S.E.; Ausin, I. CG gene body DNA methylation changes and evolution of duplicated genes in cassava. Proc. Natl. Acad. Sci. USA 2015, 112, 13729-13734. [CrossRef]

52. Bolle, C. The role of GRAS proteins in plant signal transduction and development. Planta 2004, 218, 683-692. [CrossRef]

53. Capy, P.; Gasperi, G.; Biemont, C.; Bazin, C. Stress and transposable elements: Co-evolution or useful parasites? Heredity (Edinb) 2000, 85 Pt 2, 101-106. [CrossRef]

54. Dowen, R.H.; Pelizzola, M.; Schmitz, R.J.; Lister, R.; Dowen, J.M.; Nery, J.R.; Dixon, J.E.; Ecker, J.R. Widespread dynamic DNA methylation in response to biotic stress. Proc. Natl. Acad. Sci. USA 2012, 109, E2183-E2191. [CrossRef]

55. Mosher, R.A.; Melnyk, C.W. siRNAs and DNA methylation: Seedy epigenetics. Trends Plant Sci. 2010, 15, 204-210. [CrossRef]

56. Matzke, M.A.; Mosher, R.A. RNA-directed DNA methylation: An epigenetic pathway of increasing complexity. Nat. Rev. Genet. 2014, 15, 394-408. [CrossRef]

57. Daxinger, L.; Kanno, T.; Bucher, E.; van der Winden, J.; Naumann, U.; Matzke, A.J.; Matzke, M. A stepwise pathway for biogenesis of 24-nt secondary siRNAs and spreading of DNA methylation. EMBO J. 2009, 28, 48-57. [CrossRef]

58. Ausin, I.; Feng, S.; Yu, C.; Liu, W.; Kuo, H.Y.; Jacobsen, E.L.; Zhai, J.; Gallego-Bartolome, J.; Wang, L.; Egertsdotter, U.; et al. DNA methylome of the 20-gigabase Norway spruce genome. Proc. Natl. Acad. Sci. USA 2016, 113, E8106-E8113. [CrossRef]

59. Niederhuth, C.E.; Bewick, A.J.; Ji, L.; Alabady, M.S.; Kim, K.D.; Li, Q.; Rohr, N.A.; Rambani, A.; Burke, J.M.; Udall, J.A.; et al. Widespread natural variation of DNA methylation within angiosperms. Genome Biol. 2016, 17, 194. [CrossRef]

60. Seymour, D.K.; Koenig, D.; Hagmann, J.; Becker, C.; Weigel, D. Evolution of DNA methylation patterns in the Brassicaceae is driven by differences in genome organization. PLoS Genet. 2014, 10, e1004785. [CrossRef]

61. Chan, S.W.; Henderson, I.R.; Jacobsen, S.E. Gardening the genome: DNA methylation in Arabidopsis thaliana. Nat. Rev. Genet. 2005, 6, 351-360. [CrossRef]

62. Li, X.; Zhu, J.; Hu, F.; Ge, S.; Ye, M.; Xiang, H.; Zhang, G.; Zheng, X.; Zhang, H.; Zhang, S.; et al. Single-base resolution maps of cultivated and wild rice methylomes and regulatory roles of DNA methylation in plant gene expression. BMC Genom. 2012, 13, 300. [CrossRef] [PubMed]

63. Meng, D.; Dubin, M.; Zhang, P.; Osborne, E.J.; Stegle, O.; Clark, R.M.; Nordborg, M. Limited Contribution of DNA Methylation Variation to Expression Regulation in Arabidopsis thaliana. PLoS Genet. 2016, 12, e1006141. [CrossRef] [PubMed]

64. Xu, J.; Zhou, S.; Gong, X.; Song, Y.; van Nocker, S.; Ma, F.; Guan, Q. Single-base methylome analysis reveals dynamic epigenomic differences associated with water deficit in apple. Plant Biotechnol. J. 2018, 16, 672-687. [CrossRef]

65. Mager, S.; Ludewig, U. Massive Loss of DNA Methylation in Nitrogen-, but Not in Phosphorus-Deficient Zea mays Roots Is Poorly Correlated With Gene Expression Differences. Front Plant Sci. 2018, 9, 497. [CrossRef]

66. Zhang, B.; Tieman, D.M.; Jiao, C.; Xu, Y.; Chen, K.; Fei, Z.; Giovannoni, J.J.; Klee, H.J. Chilling-induced tomato flavor loss is associated with altered volatile synthesis and transient changes in DNA methylation. Proc. Natl. Acad. Sci. USA 2016, 113, 12580-12585. [CrossRef] [PubMed]

67. Song, Y.; Ji, D.; Li, S.; Wang, P.; Li, Q.; Xiang, F. The dynamic changes of DNA methylation and histone modifications of salt responsive transcription factor genes in soybean. PLoS ONE 2012, 7, e41274. [CrossRef]

68. Huang, K.L.; Ma, G.J.; Zhang, M.L.; Xiong, H.; Wu, H.; Zhao, C.Z.; Liu, C.S.; Jia, H.X.; Chen, L.; Kjorven, J.O.; et al. The ARF7 and ARF19 Transcription Factors Positively Regulate PHOSPHATE STARVATION RESPONSE1 in Arabidopsis Roots. Plant Physiol. 2018, 178, 413-427. [CrossRef]

69. Mager, S.; Schonberger, B.; Ludewig, U. The transcriptome of zinc deficient maize roots and its relationship to DNA methylation loss. BMC Plant Biol. 2018, 18, 372. [CrossRef]

70. Boyko, A.; Kathiria, P.; Zemp, F.J.; Yao, Y.; Pogribny, I.; Kovalchuk, I. Transgenerational changes in the genome stability and methylation in pathogen-infected plants: (Virus-induced plant genome instability). Nucleic Acids Res. 2007, 35, 1714-1725. [CrossRef]

71. Iwasaki, M.; Paszkowski, J. Epigenetic memory in plants. EMBO J. 2014, 33, 1987-1998. [CrossRef] 
72. Garg, R.; Narayana Chevala, V.; Shankar, R.; Jain, M. Divergent DNA methylation patterns associated with gene expression in rice cultivars with contrasting drought and salinity stress response. Sci. Rep. 2015, 5, 14922. [CrossRef]

73. Zhang, D.; Zhang, H.; Chu, S.; Li, H.; Chi, Y.; Triebwasser-Freese, D.; Lv, H.; Yu, D. Integrating QTL mapping and transcriptomics identifies candidate genes underlying QTLs associated with soybean tolerance to low-phosphorus stress. Plant Mol. Biol. 2017, 93, 137-150. [CrossRef] [PubMed]

74. Porebski, S.; Bailey, L.G.; Baum, B.R. Modification of a CTAB DNA extraction protocol for plants containing high polysaccharide and polyphenol components. Plant Mol. Biol. Rep. 1997, 15, 8-15. [CrossRef]

75. Xi, Y.; Li, W. BSMAP: Whole genome bisulfite sequence MAPping program. BMC Bioinform. 2009, 10, 232. [CrossRef]

76. Zhang, H.; Yang, Y.; Sun, C.; Liu, X.; Lv, L.; Hu, Z.; Yu, D.; Zhang, D. Up-regulating GmETO1 improves phosphorus uptake and use efficiency by promoting root growth in soybean. Plant Cell Environ. 2020. [CrossRef] [PubMed]

77. Kim, D.; Pertea, G.; Trapnell, C.; Pimentel, H.; Kelley, R.; Salzberg, S.L. TopHat2: Accurate alignment of transcriptomes in the presence of insertions, deletions and gene fusions. Genome Biol. 2013, 14, R36. [CrossRef]

78. Trapnell, C.; Roberts, A.; Goff, L.; Pertea, G.; Kim, D.; Kelley, D.R.; Pimentel, H.; Salzberg, S.L.; Rinn, J.L.; Pachter, L. Differential gene and transcript expression analysis of RNA-seq experiments with TopHat and Cufflinks. Nat. Protoc. 2012, 7, 562-578. [CrossRef]

79. Li, R.; Yu, C.; Li, Y.; Lam, T.W.; Yiu, S.M.; Kristiansen, K.; Wang, J. SOAP2: An improved ultrafast tool for short read alignment. Bioinformatics 2009, 25, 1966-1967. [CrossRef]

(C) 2020 by the authors. Licensee MDPI, Basel, Switzerland. This article is an open access article distributed under the terms and conditions of the Creative Commons Attribution (CC BY) license (http://creativecommons.org/licenses/by/4.0/). 